\title{
Restriction of non-coding elements in the thymus by Aire
}

Shahan Mamoor ${ }^{1}$, MS

1Thomas Jefferson School of Law

San Diego, CA 92101

correspondence: mamoorsk@tjsl.edu

Aire is a unique transcriptional molecule that functions in immunological tolerance by promoting promiscuous expression of tissue-restricted genes. The expression of these otherwise tissue-restricted genes in the medullary epithelial cells of the thymus (mTECs) allows the presentation of self antigens to "teach" cells of the immune system what it means to be "self". To understand the role of Aire in shaping the mTEC transcriptome, I performed global differential gene expression analysis of wild-type mTECs and mTECs deficient in Aire. I identified nineteen non-coding molecules whose expression were significantly modulated by the absence of Aire. These included pseudogenes, antisense RNA, small nucleolar and small nuclear RNA, and long noncoding RNA. This finding reveals that Aire controls the expression of both coding and non-coding genes, and further suggests that non-coding RNA might play a role in Aire's instruction of immunological tolerance.

Keywords: Aire, non-coding RNA, IncRNA, snoRNA, snRNA, pseudogene, antisense RNA, medullary thymic epithelial cell (mTEC). 


\section{Introduction}

Distinguishing the self from non-self is essential for the precise function of the immune system. In addition to the ability to discriminate between foreign and self molecules, the ability to tolerate the molecules, cells and tissues of the self is critical for the prevention of autoimmune disease. In order to teach developing lymphocytes what it means to be "self", medullary epithelial cells of the thymus, or mTECs express genes that code for antigens whose expression is otherwise restricted to tissues in the periphery (tissue-restricted antigens). This promiscuous expression of tissue-restricted antigens by Aire and their subsequent presentation is a mechanism by which tolerance of tissue-specific antigens is established centrally in the thymus.

Accordingly, humans that have mutations in Aire experience autoimmune polyendocrinopathy candidiasis ectodermal dystrophy, or APECED (also known as autoimmune polyendocrine syndrome type 1 (APS-1)) (2). These patients possess multi-organ autoimmune pathology and a classical triad of mucocutaneous candidiasis, autoimmune adrenocortical insufficiency and autoimmune hypoparathyroidism. Over 42 separate mutations affecting Aire have been discovered in patients with APECED/ APS-1 (2); the underlying mechanism by which mutations in Aire lead to APECED is unknown.

To understand that transcriptional changes that accompany Aire expression in the mTECs, I performed global gene expression profiling and compared the transcriptomes of mTECs with high expression of the class II major histocompatibility complex (mTEChi) that possess and lack Aire (1). Surprisingly, of the most differentially expressed genes between Aire -/- and Aire +/+ mTECs, I identified nineteen non-coding 
RNA. These non-coding species include long and long intergenic ncRNA, small nuclear RNA, small nucleolar RNA, pseudogenes and antisense RNA. The discovery that noncoding RNA represent a major element of the Aire-induced transcriptome is unprecedented and suggests that Aire may function in immunological tolerance via noncoding mechanisms.

\section{Methods}

The GEO2R platform was utilized to compare samples from dataset GSE102527 (1) and identify the two hundred and fifty most differentially regulated transcripts between medullary thymic epithelial cells from wild-type and Aire knockout mice ( $n=2$ samples each). No adjustment was made to $p$-values, the data was not logtransformed in any manners. Unpaired Welch's t-test with correction was performed for each comparison to determine the statistical significance of the difference between the raw sample values (PRISM 8).

\section{Results}

I mined a pre-existing transcriptome dataset of mTECs expressing high levels of the class II major histocompatibility complex (mTEChi), both wild-type and Aire-deleted (1), to determine the most significant transcriptional changes induced by the absence of Aire. I identified nineteen non-coding transcripts that were among the most differentially expressed genes when comparing the transcriptomes of Aire +/+ and Aire -/- mTEChi. Ten of the non-coding transcripts were up-regulated in the absence of Aire while nine of the non-coding transcripts were down-regulated in its absence. 
Four of the transcripts were identified to be pseudogenes (Figure 1), nine were identified as long or long intergenic non-coding RNA (IncRNA or lincRNA, respectively; Figure 2), three were identified to be small RNA of either nuclear (snRNA) or nucleolar (snoRNA) types (Figure 3), and three were identified as antisense transcripts (Figure 4).

The Aly/Ref gene encodes machinery involved in the nuclear export of mRNA. The Aly/Ref pseudogene transcript, encoded on Chromosome 1 was one of the most differentially expressed genes when comparing the transcriptomes of mTEC hi with and without Aire. The Aly/Ref pseudogene is expressed at significantly lower levels in Aire-/mTEChi (Figure $1 ; p=0.0009$ ). Expression of the Aly/Ref pseudogene is nearly ablated in mTEChi lacking Aire.

GM7609 is a non-coding RNA and predicted pseudogene transcript encoded on Chromosome 1 that is annotated by NCBI as Sp100-rs and also among the genes most differentially expressed in Aire-/- mTEChi compared to their wild-type counterparts. ncRNA GM6709 was expressed at higher levels in Aire -/- mTEChi (Figure 1; $p=0.0339$ ). Gm9782/NM_001270426 is a predicted pseudogene transcript encoded on Chromosome 3. Pseudogene Gm9782 was expressed at significantly lower levels in

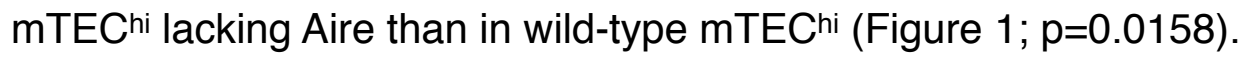

ENSMUST00000070435 is a pseudogene transcript encoded on Chromosome 10 and also known as fatty acid binding protein 3, muscle and heart. Pseudogene transcript ENSMUST00000070435 was expressed at significantly lower levels in Aire-/-

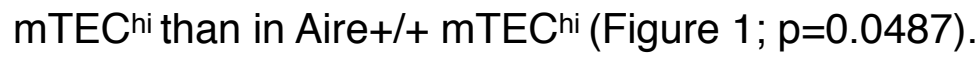

NR_003647/Gm5039, encoded on Chromosome 12, is a 1641 bp IncRNA and one of the most differentially expressed genes in mTEChi lacking Aire. IncRNA 
NR_003647/Gm5039 was expressed at significantly lower levels in Aire -/- mTEChi (Figure 2; $p=0.0217$ ).

Gm9999, encoded by a gene on Chromosome 7, was also one of the most differentially expressed transcripts between the transcriptomes of mTEChi and Aire -/mTEChi. While MGI and Ensemble gene models have annotated this gene as proteincoding, NCBI has designated Gm9999 as a non-coding RNA. Gm9999 was expressed in significantly lower quantities in Aire-deleted mTEChi (Figure 2; $p=0.0131$ ).

Transcript NR_040392, or 1700063D05Rik, is a long non-coding RNA encoded by a gene on Chromosome 9. Data from the mouse ENCODE project indicates that IncRNA NR_040392 is expressed at highest levels in the testis and cerebellum. IncRNA NR_040392 was expressed at significantly lower levels in mTEChi lacking Aire (Figure 2; $\mathrm{p}=0.0211)$.

Gm5/NR_024513 is a 470 bp long non-coding RNA encoded on Chromosome 5. IncRNA Gm5/NR_024513 was expressed at significantly lower levels in Aire -/- mTEChi (Figure 2; $p=0.0028$ ).

ENSMUST00000147509, also known as GM15391, is a 257 basepair long intergenic non-coding RNA (lincRNA) and its gene, located on Chromosome 1 is one of the most differentially expressed when comparing the transcriptomes of mTEChi that do or do not express Aire. lincRNA ENSMUST00000147509/GM15391 is expressed at significantly higher levels in mTEChi in the absence of Aire (Figure 2; $\mathrm{p}=0.0390$ ).

A930001A20Rik / AK019124 is a IncRNA encoded by a gene on Chromosome 3 and was among the most differentially expressed genes when comparing the global gene expression profiles of mTEChi sufficient and deficient for Aire. lincRNA 
A930001A20Rik was expressed at significantly higher levels in Aire-deleted mTEChi (Figure 2; $p=0.0357$ ).

NR_033483, a 522 bp IncRNA also known as U90926, is encoded on Chromosome 5 and was one of the most differentially expressed genes when comparing the transcriptomes of Aire -/- and Aire +/+ mTEChi, NCBI annotates NR_033483 as a putative TNF-resistance related protein mRNA. NR_033483 was expressed in Aire -/- mTEChi at lower levels than in Aire, and this decreased expression approached statistical significance (Figure 2; $p=0.0514$ ).

NR_046041, also known as GM11240, is a 759 base pair long non-coding RNA encoded on Chromosome 4. IncRNA NR_046041 was expressed at higher levels in Aire -/- mTEChi when compared to Aire +/+ mTEChi and this increased expression trended towards statistical significance (Figure 2; $p=0.0651$ ).

NR_040328 is an 881 bp long-non coding RNA encoded by a gene located on Chromosome 18. IncRNA NR_040328 was among the genes most differentially expressed in Aire-deleted mTEChi and expressed at significantly higher levels in Airedeleted mTEChi than in wild-type mTEChi (Figure 2; $p=0.0123$ ).

NR_003647/Gm5039, encoded by a gene located on Chromosome 12, is a 1641 bp IncRNA and one of the most differentially expressed genes in mTEChi lacking Aire. IncRNA NR_003647/Gm5039 was expressed at significantly lower levels in Aire -/-

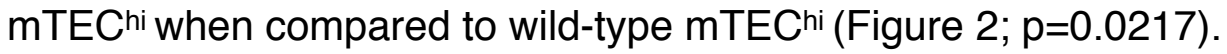

ENSMUST00000083030 snRNA is a 105 bp small nuclear RNA (snRNA) encoded by a gene located on Chromosome 2. snRNA ENSMUST00000083030 was among the genes most differentially expressed in Aire-/- mTEChi when compared to 
Aire +/+ mTEChi and expressed at significantly higher levels in Aire-/- mTEChi (Figure 3; $p=0.0205)$.

snoRNA ENSMUST00000158627, or Gm25715-201 is a 125 bp small nucleolar RNA encoded by a gene on Chromosome 16. snoRNA ENSMUST00000158627/ Gm25715 was expressed at significantly higher levels in mTEChi lacking Aire (Figure 3; $p=0.0001)$.

snoRNA ENSMUST00000104164, encoded by a gene located on Chromosome 6 , was also one of the most differentially expressed genes when comparing the transcriptomes of mTEChi with and without Aire. snoRNA ENSMUST00000104164 was expressed at significantly higher levels in Aire-deleted mTEChi (Figure 3; $p=0.0197$ ).

While only two snoRNA were identified among the genes whose expression was most differentially regulated in mTECs that lack Aire, the expression of both increased significantly after deletion of Aire. This might suggest that Aire restricts the expression of snoRNA in cells of the thymus that possess high expression of the class II major histocompatibility molecule (MHC Class II).

Antisense 2510003D118Rik corresponds to the opposite strand transcript from the ankyrin repeat and SOCS box-containing 17 gene located on Chromosome 3. Antisense 2510003D118Rik was expressed at significantly lower levels in Aire -/-

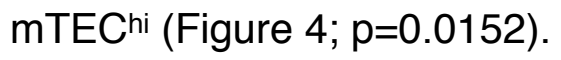

ENSMUST00000153522, also known as Gm15600, is encoded by a gene located on Chromosome 9 and measures 856 basepairs long. Antisense transcript ENSMUST00000153522/Gm15600 (or Gm15600-AS) was among the most differentially 
expressed transcripts between Aire +/+ and Aire -/- mTEChi. Gm15600-AS was produced at significantly higher levels in Aire -/- mTEChi (Figure 4; $p=0.0134$ ).

Finally, ENSMUST00000138402, also known as Gm12089 is an antisense transcript encoded on Chromosome 11 and was among the genes most differentially expressed between mTEChi that possess a functional copy of the Aire gene and those that do not. Antisense Gm12089 was expressed at higher levels in Aire -/- mTEChi and this higher expression approached but did not reach statistical significance (Figure 4; $p=0.0611)$.

\section{Discussion}

Tolerance occurs in the periphery and centrally; Aire is a critical molecule for the execution of central tolerance and loss of Aire function results in autoimmune disease in humans $(1,2)$. To understand the transcriptional effects of Aire on a global scale, I compared the transcriptomes of thymic medullary epithelial cells possessing or lacking Aire (1). I found that nineteen of the two hundred and fifty genes whose expression was most differentially regulated between mTECs deficient and sufficient for Aire were noncoding RNA transcripts. These included four pseudogenes, nine long non-coding RNA, three small nuclear or nucleolar RNA and three antisense RNA transcripts. These findings demonstrate that non-coding RNA are represented among the set of transcripts most differentially expressed in Aire -/- mTECs, and suggests that Aire is involved in the transcriptional control of non-coding RNAs. Future work is required to determine the role that non-coding RNA play (if any) in the establishment of tolerogenic mechanisms. 


\section{References}

1. Koh, A.S., Miller, E.L., Buenrostro, J.D., Moskowitz, D.M., Wang, J., Greenleaf, W.J., Chang, H.Y. and Crabtree, G.R., 2018. Rapid chromatin repression by Aire provides precise control of immune tolerance. Nature immunology, 19(2), p.162.

2. Heino, M., Peterson, P., Kudoh, J., Shimizu, N., Antonarakis, S.E., Scott, H.S. and Krohn, K., 2001. APECED mutations in the autoimmune regulator (AIRE) gene. Human mutation, 18(3), pp.205-211. 
Figure 1: Pseudogenes are among the transcripts most differentially expressed by mTECs deficient in Aire.

Gm9782 pseudogene

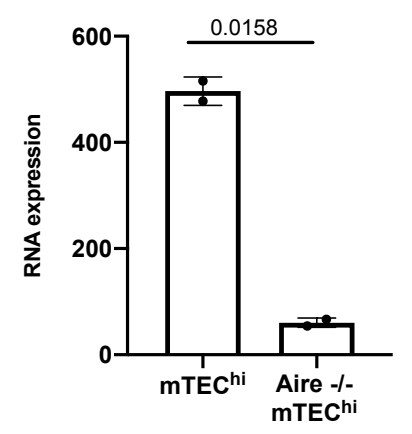

ENSMUST00000070435 pseudogene

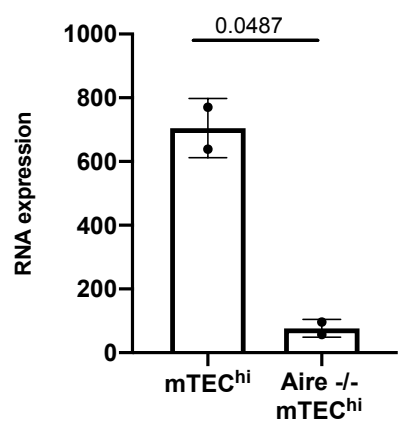

Aly/REF pseudogene

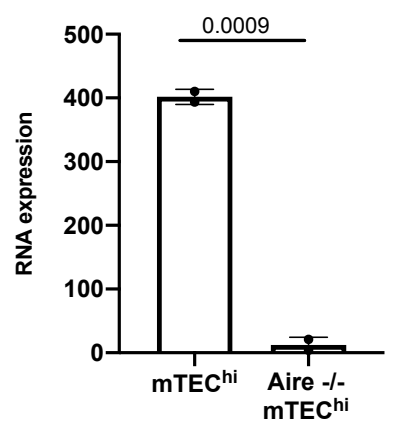

Gm7609 pseudogene

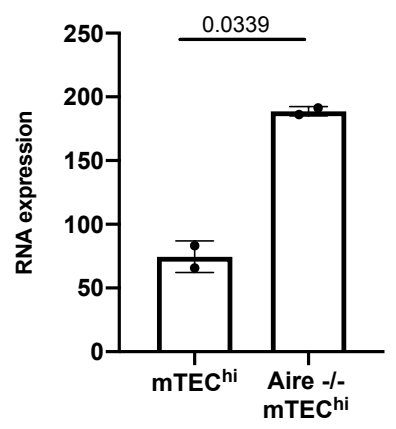

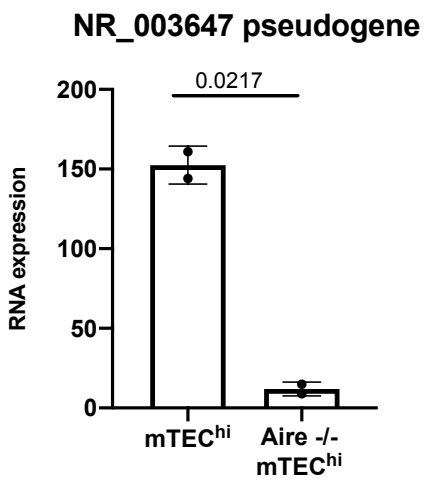

The mean RNA expression value of the specified non-coding transcripts in mTEChi and in Aire-deleted mTEChi $(n=2$, each) is depicted in graphical form with the error bars representing the standard deviation. The exact $p$-value of the comparison between the two mean RNA expression values, determined by an unpaired Welch's t-test, is indicated on each respective graph. 
Figure 2: Long and long intergenic non-coding RNA are among the transcripts most differentially expressed by mTECs deficient in Aire.
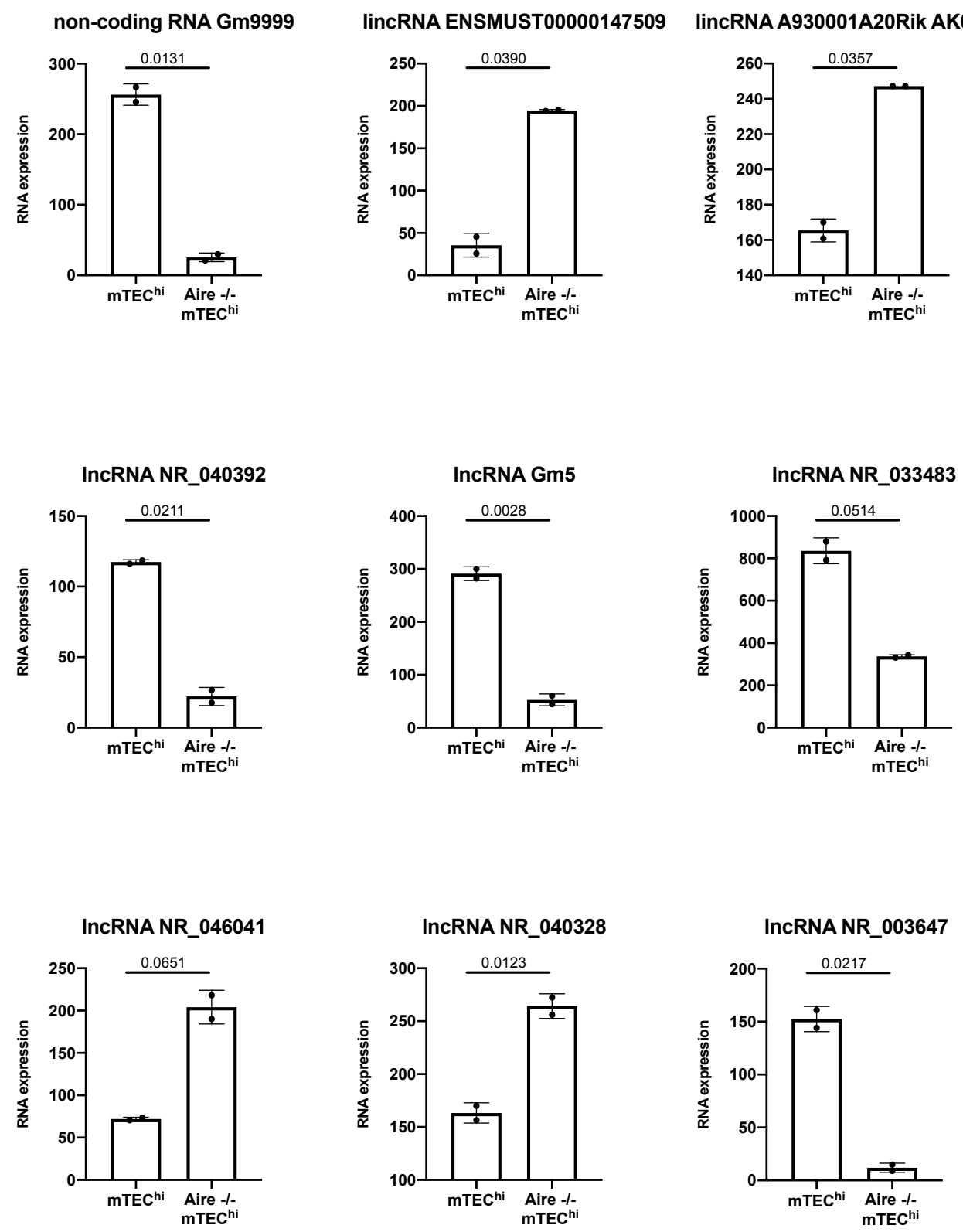

The mean RNA expression value of the specified non-coding transcripts in mTEChi and in Aire-deleted mTEChi $(n=2$, each) is depicted in graphical form with the error bars representing the standard deviation. The exact $p$-value of the comparison between the two mean RNA expression values, determined by an unpaired Welch's t-test, is indicated on each respective graph. 
Figure 3: Small nucleolar and small nuclear non-coding RNA are among the transcripts most differentially expressed by mTECs deficient in Aire.

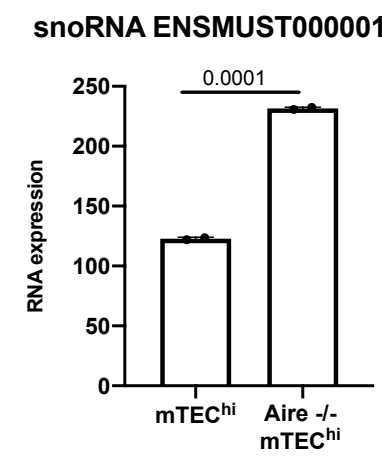

snoRNA ENSMUST00000104164 snoRNA
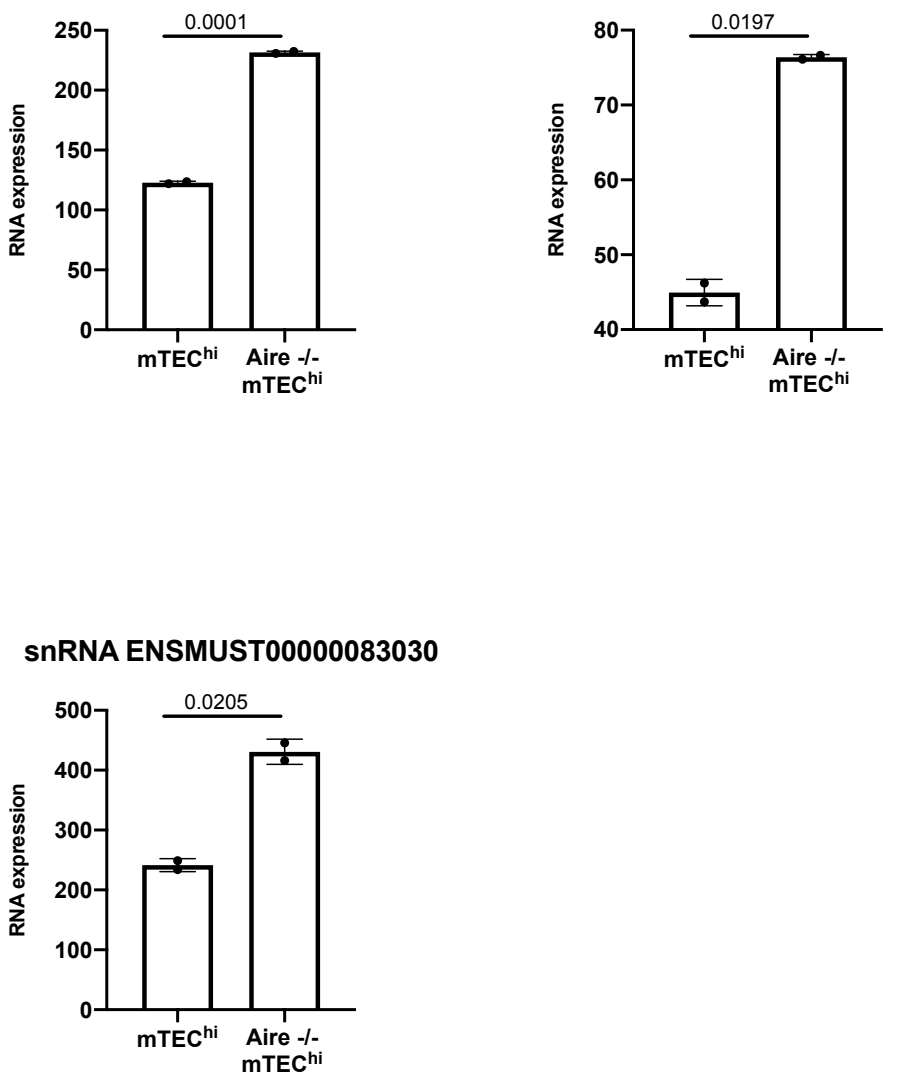

The mean RNA expression value of the specified non-coding transcripts in mTEChi and in Aire-deleted mTEChi $(n=2$, each) is depicted in graphical form with the error bars representing the standard deviation. The exact $p$-value of the comparison between the two mean RNA expression values, determined by an unpaired Welch's t-test, is indicated on each respective graph. 
Figure 4: Antisense non-coding RNA are among the transcripts most differentially expressed by mTECs deficient in Aire.
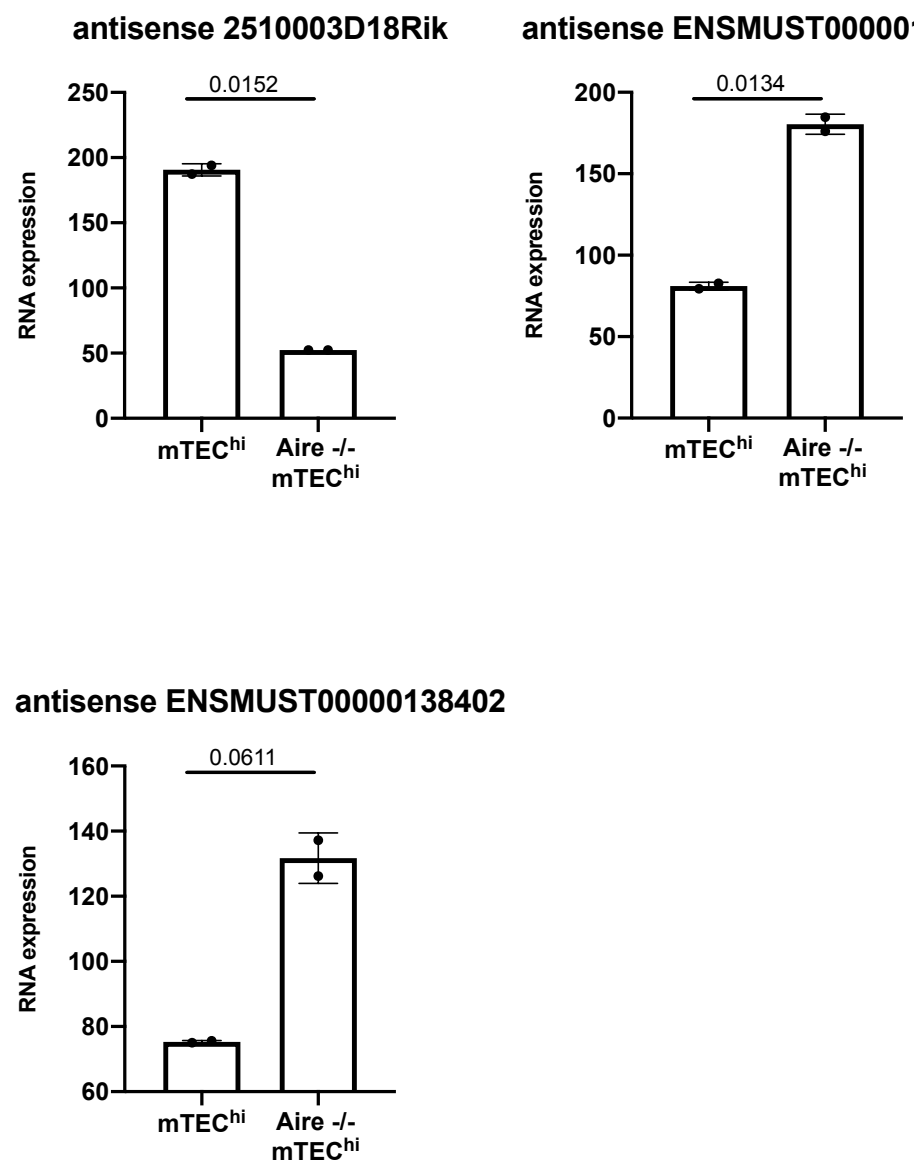

The mean RNA expression value of the specified non-coding transcripts in mTEChi and in Aire-deleted mTEChi $(n=2$, each) is depicted in graphical form with the error bars representing the standard deviation. The exact $p$-value of the comparison between the two mean RNA expression values, determined by an unpaired Welch's t-test, is indicated on each respective graph. 